\title{
On the Temperature Dependent Mechanical Response of Dynamically-loaded Shear- dominated Adhesive Structures
}

\author{
Borja Erice $^{1,2^{*}}$, Maria Lißner ${ }^{3}$, Jan Wittig ${ }^{4}$, Andreas Hornig ${ }^{4}$, Maik Gude $^{4}$ and Nik Petrinic ${ }^{3}$ \\ ${ }^{1}$ Department of Mechanics and Industrial Production, Mondragon Unibertsitatea, \\ Loramendi 4, 20500 Mondragon, Spain \\ ${ }^{2}$ IKERBASQUE, Basque Foundation for Science, Bilbao, Spain \\ ${ }^{3}$ Department of Engineering Science, University of Oxford, Parks Road, OX1 3PJ Oxford, UK \\ ${ }^{4}$ Institute of Lightweight Engineering and Polymer Technology (ILK), \\ Technische Universität Dresden, Holbeinstraße 3, Dresden 01307, Germany
}

\begin{abstract}
A mode II mechanical characterisation of the adhesive joints is performed testing shear lap joint specimens in a Split Hopkinson Tensile Bar (SHTB), equipped with a temperature chamber. The experimentallyobtained traction-separation curves were used to develop a Cohesive Zone Model (CZM) capable of representing the strain-rate and temperaturedependent mechanical response of the adhesive joints. To validate the model, End Notch Flexure (ENF) multi-material specimens made from titanium and carbon fibre reinforced polymer composite laminates were tested at different temperatures using a Split Hopkinson Pressure Bar setup with an in-house made temperature chamber. The finite element (FE) simulations of such tests employing the developed CZM showed the model's ability to accurately predict the adhesive joints' failure as well as to understand the failure sequence of multi-material adhesive joint combinations.
\end{abstract}

\section{Introduction}

In many industrial sectors including, but not limited to the transportation sector, structures are subjected to environments of different nature, i.e. severe loading rate changes, bird strikes or hail impact on aircraft structures or even temperature or humidity changes. Those changes rapidly or gradually applied, have to be considered in the design stages so that they won't negatively affect the mechanical performance of the materials and load bearing structures. This especially is of utmost importance when considering polymeric-based adhesives used to join different materials since they tend to depend largely on the strain-rate and on the temperature. The effect of the temperature under quasi-static loading conditions on the mechanical response of these materials has been extensively investigated [1]. However, their behaviour at different temperatures and loading rate regimes is still limited, which is directly related to the complexity of the experimental setup and its ability to provide reasonable

* Corresponding author: berice@mondragon.edu 
results. Although, further investigation is necessary, an initial attempt is made to obtain reliable data that allows predicting the shear-dominated failure behaviour of adhesively bonded structures at different temperatures and high loading rates.

\section{Single lap shear experiments (mode II)}

The mode II mechanical characterisation of the thermosetting epoxy resin AF 163-2OST from Scotch-Weld ${ }^{\mathrm{TM}}$ adhesive joints was performed testing shear lap joint specimens in a Split Hopkinson Tensile Bar (SHTB), described in detail in [2], equipped with a temperature chamber designed specifically for this study (see Figure 1(a)). The tests were conducted for three adhesive layer thicknesses, $0.1,0.3$ and $0.5 \mathrm{~mm}$ and for three temperatures 25,50 and $100{ }^{\circ} \mathrm{C}$. The fixtures (see Figure $1(\mathrm{~b})$ ) and the SHTB components, $16 \mathrm{~mm}$ diameter input and output bars, were made out of $\mathrm{Ti}-6 \mathrm{Al}-4 \mathrm{~V}$.

The specimen shear stress histories were directly calculated from the transmitted strain wave $\left(\varepsilon_{t}\right)$ signals given by the strain gauges attached to the output bar as $\sigma[t]=A_{b} E_{b} \varepsilon_{t}[t] / A_{0}$, where $E_{b}$ and $A_{b}$ are the elastic modulus and the cross-section area of the bar and $A_{0}$ is the initial cross-section area of the specimen. The displacement separation histories $u[t]$ between the bonded surfaces was computed post-processing the $924 \times 768 \mathrm{px}^{2}$ resolution images taken at $500 \mathrm{kfps}$ with a Kirana high speed camera using the GOM Aramis commercial Digital Image Correlation (DIC) software. The stress-displacement curves for the three temperatures and thicknesses of $0.1,0.3$ and $0.5 \mathrm{~mm}$ are shown in Figure 1 (c), (d) and (e) respectively. The adhesive showed a clear temperature dependency, exhibiting a decrease in strength and an increase in the fracture energy.

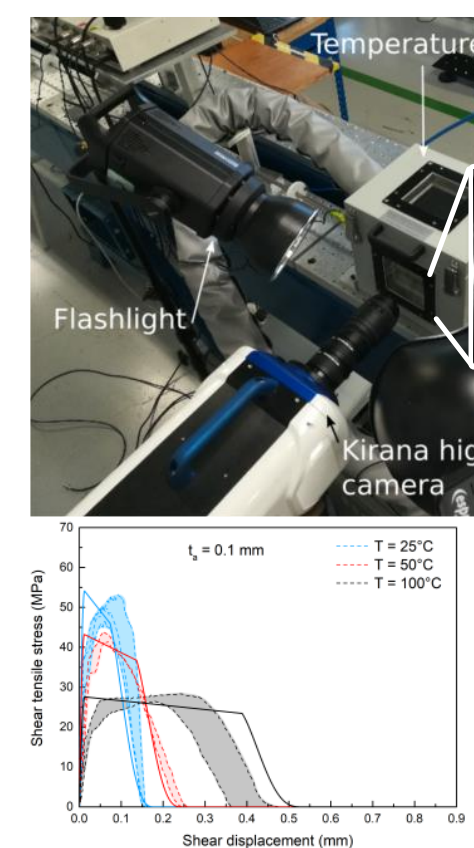

(c)

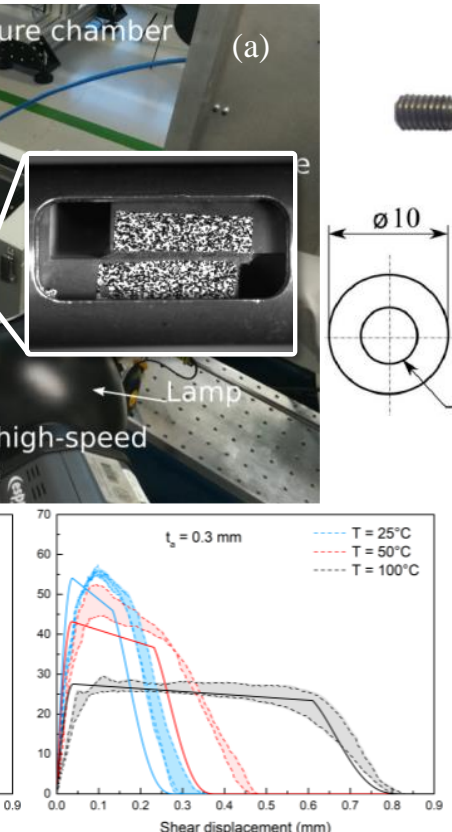

(d)

(b)

Figure 1. Experimental setup (a) and fixtures (b) employed to test the single lap shear joint specimens at different temperatures. Experimental vs. numerical high strain rate traction-separation curves of mode II specimens for thicknesses of 0.1 (c), 0.3 (d) and $0.5 \mathrm{~mm}$ (e) with varying temperatures. 


\section{Cohesive zone model}

The CZM is an extension of the rate-dependent tri-linear traction-separation law previously presented in [2], that takes into account the adhesive thickness as well as the void volume fraction variation across such thickness. The traction and separation vectors, $\mathbf{t}$ and $\boldsymbol{\delta}$, are defined as,

$$
\mathbf{t}=\left\{\begin{array}{ll}
t_{n} & t_{s}
\end{array}\right\}^{T} \text { and } \boldsymbol{\delta}=\left\{\begin{array}{ll}
\delta_{n} & \delta_{s}
\end{array}\right\}^{T}=\left\{\begin{array}{ll}
\left\langle u_{1}\right\rangle & \sqrt{u_{2}^{2}+u_{3}^{2}}
\end{array}\right\}^{T}
$$

where $n$ and $s$ indicate normal and shear directions and $u_{1}, u_{2}$ and $u_{3}$ are the displacement vector components in the normal and tangential directions. The traction-separation law reads:

$$
\mathbf{t}=(1-d) \mathbf{K} \cdot \boldsymbol{\delta}
$$

where $d$ is the damage parameter, which will be defined later. The $2 \times 2$ diagonal stiffness matrix components are $K_{n}=E / t$ and $K_{s}=G / t$ that are calculated dividing the elastic and shear moduli over the thickness $t$. The yielding traction corresponding to the plateau stage (see Figure 2(a)) is given by,

$$
T_{i}\left[\dot{\varepsilon}_{i}, t\right]=T_{0, i}\left(1+k_{T, i} \ln \left[\dot{\varepsilon}_{i} / \dot{\varepsilon}_{0}\right]\right)\left(1-f_{V}[t]\right)
$$

where $\dot{\varepsilon}$ is the strain rate, $k_{T}$ is the rate-dependent coefficient, $f_{v}=f_{0} t^{-\alpha}$ is the void volume fraction variation with the adhesive thickness and $\alpha$ is a material constant fitted from the experimental data. Henceforth, the subindex $i=n, s$ will be adopted to indicate normal and shear directions and the subindex 0 will indicate a reference magnitude. The separations shown in Figure 2(a) after the onset of yielding are given by,

Separation at onset of yielding, $\delta_{i, 1}=T_{i} / K_{i}$

$$
\text { Separation at damage initiation } \delta_{i, 2}=\delta_{i, 1}+\frac{2 G_{C, i} P_{i}}{T_{i}\left(1+\gamma_{i}\right)}
$$

$$
\text { Separation at failure, } \delta_{i, f}=\delta_{i, 1}+\delta_{i, 2}+\frac{2 G_{C, i}}{T_{i} \gamma_{i}}-\frac{\delta_{i, 2}+\gamma_{i}\left(\delta_{i, 2}-\delta_{i, 1}\right)}{\gamma_{i}}
$$

The parameter $\gamma$ is the softening parameter or plateau decrease, $G_{C}$ is the total energy dissipated during the crack opening process and $P$ is the ratio between the plateau and the total energies. The total and partial energies are rate-dependent and are defined given by the following expressions,

$$
G_{C, i}\left[\dot{\varepsilon}_{i}, t\right]=G_{0, i}\left(1-k_{G, i} \ln \left[\dot{\varepsilon}_{i} / \dot{\varepsilon}_{0}\right]\right)\left(1-f_{V}[t]\right)\left(1-t_{0} / t\right) \text { and } P_{i}\left[\dot{\varepsilon}_{i}\right]=P_{0, i}\left(\dot{\varepsilon}_{i} / \dot{\varepsilon}_{0}\right)^{p_{i}}
$$

where $k_{G}$ and $p$ are a rate-dependent coefficient and exponent respectively. The modemixity is assumed to follow a quadratic criterion for the onset of yielding and linear for the rest of the stages [2], and therefore the mixed mode displacement $\delta_{m}$ is,

$$
\delta_{m 1}=\delta_{n 1} \delta_{s 1} \sqrt{\frac{1+\beta^{2}}{\delta_{s 1}^{2}+\left(\beta \delta_{n 1}\right)^{2}}} \quad \text { and } \quad \delta_{m, j}=\delta_{n, j} \delta_{s, j} \frac{\sqrt{1+\beta^{2}}}{\left(\delta_{s, j}+\beta \delta_{n, j}\right)} \quad \text { for } \quad j=2, f
$$

where $\beta=\delta_{s, j} / \delta_{n, j}$ is the mixed mode ratio. For the sake of completion the mixed mode stiffness is given,

$$
K_{m}=\sqrt{\frac{K_{n}^{2}+\left(\beta K_{s}\right)^{2}}{1+\beta^{2}}}
$$

The damage parameter can be now fully defined as, 


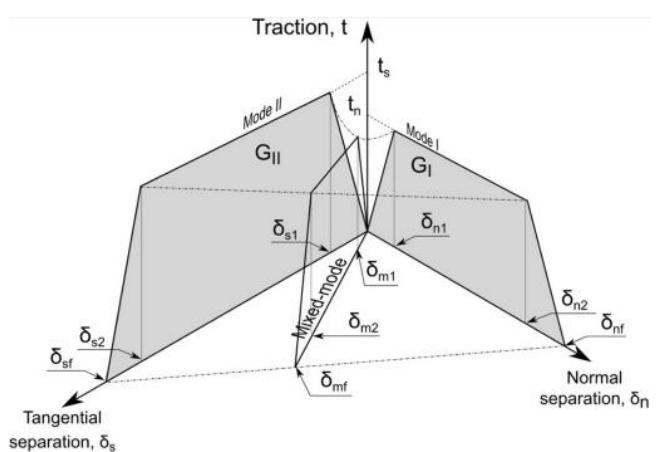

(a)

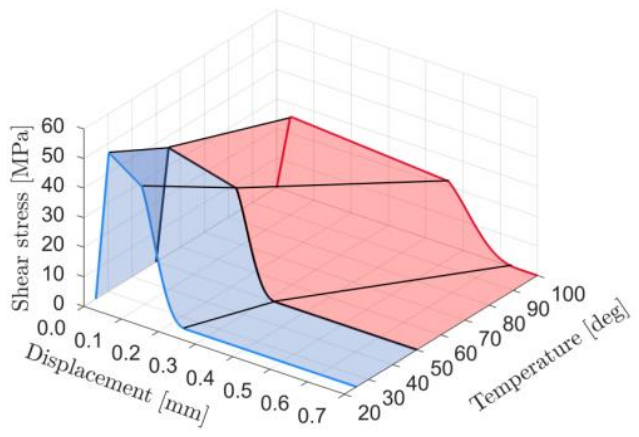

(b)

Figure 2. (a) Illustration of the CZM traction-separation law. (b) Temperature tabulated calibrated traction separation law for the AF 163-2OST adhesive.

$$
d=\left\{\begin{array}{cc}
1-\frac{\delta_{m 1}}{\delta_{m}}\left\{1+\frac{\left(\gamma_{m}-1\right)\left(\delta_{m}-\delta_{m 1}\right)}{\left(\delta_{m 2}-\delta_{m 1}\right)}\right\} & , \delta_{m} \leq \delta_{m 1} \\
1-\left\{\frac{\gamma_{m} \delta_{m 1}}{\delta_{m}} \frac{\left(\delta_{m f}-\delta_{m}\right)}{\left(\delta_{m f}-\delta_{m 2}\right)}\right\}\left\{2\left(\frac{\delta_{m}-\delta_{m 2}}{\delta_{m f}-\delta_{m 2}}\right)^{3}-3\left(\frac{\delta_{m}-\delta_{m 2}}{\delta_{m f}-\delta_{m 2}}\right)^{2}+1\right\} & , \delta_{m 1}<\delta_{m} \leq \delta_{m 2} \\
1 & , \delta_{m f}<\delta_{m} \leq \delta_{m f}
\end{array}\right.
$$

being $\gamma_{m}$ the mixed mode plateau decrease,

$$
\gamma_{m}=\sqrt{\frac{\gamma_{n}^{2}+\left(\beta \gamma_{s}\right)^{2}}{1+\beta^{2}}}
$$

The material model constants were calibrated combining experimental results from quasistatic and medium and high rate mode I, II and I/II tensile tests with X-Ray tomography analysis [2]. The temperature effect was calibrated assuming the same void volume fraction distribution as that seen in the room temperature specimens, and only employing the experimental data shown in section 2 . The effect of the temperature on the peak stresses and the total and partial energies was implemented in a tabular way (calibrated data reported in Table 1) allowing for linear interpolation between the target temperatures, in this case, $25^{\circ} \mathrm{C}$, $50{ }^{\circ} \mathrm{C}$ and $100^{\circ} \mathrm{C}$ (see Figure 2(b)). Numerical simulations of the single-lap shear specimens following the numerical setup and recommendations as those described in [2] were performed in the ABAQUS/Explicit FE commercial solver. The numerical predictions were in good agreement with the experimental mechanical responses as shown in Figure 1 (c), (d) and (e).

\section{Validation with ENF experiments}

Multi-material ENF specimens bonded with $0.3 \mathrm{~mm}$ thickness adhesive layer were tested at 25, 50 and $100{ }^{\circ} \mathrm{C}$ in a SHPB system configured as shown in [3] and instrumented as in [7]. The experimental setup is illustrated in Figure 3 . The adherent in contact with the loading pin was $\mathrm{Ti}-6 \mathrm{Al}-4 \mathrm{~V}$ and the adherent in contact with the support was made out of IM7/8552 $0.125 \mathrm{~mm}$ prepregs plies forming a laminate with a [45/-45/0/0/45/0/0/0/0/0/-45/0/45/0/-

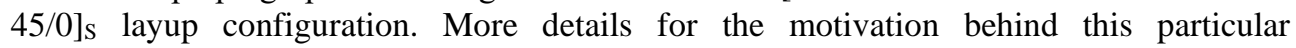
configuration can be found in [4]. A Photron SA-5 camera was set to record $716 \times 624 \mathrm{px}^{2}$ images of the displacement of the loading pin at $150 \mathrm{kfps}$ and a Kirana camera focused on the crack area recorded $924 \times 768 \mathrm{px}^{2}$ resolution images at $200 \mathrm{kfps}$. 
Table 1. Calibrated model parameters of the traction separation law

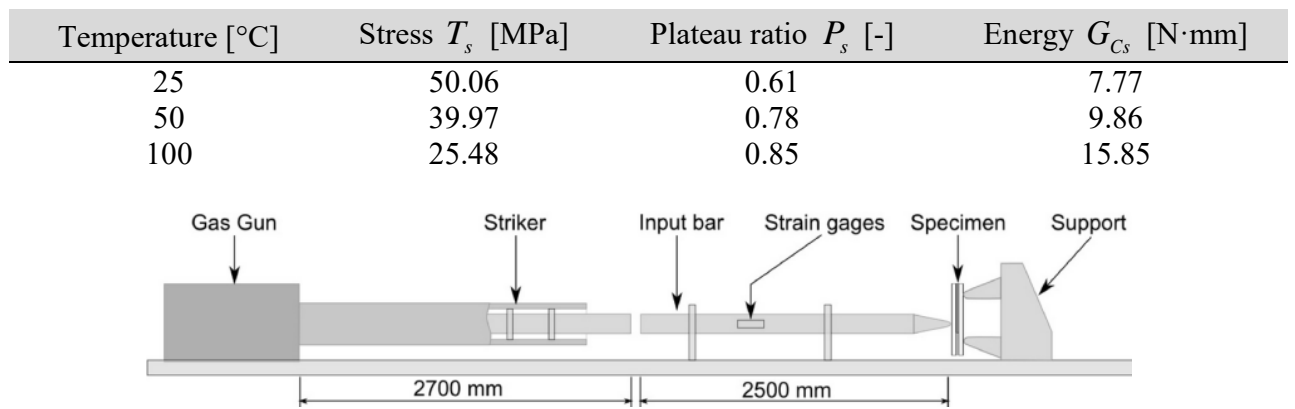

Figure 3. Geometry, dimensions and configuration of the SHPB ENF tests.

From the one dimensional elastic wave propagation theory the force histories can be obtained if properly spaced strain gauge stations are glued on the input bar [5]. Using the same approach as that used in [3], $F[t]=A_{b} E_{b}\left(\varepsilon_{i}[t]-\varepsilon_{r}[t]\right)$ where $\varepsilon_{i}$ and $\varepsilon_{r}$ are the incident and reflected strain waves. The displacement history of the loading pin was obtained from the DIC analysis of the images taken with the Photron camera. The resulting measured forcedisplacement curves can oftentimes over or under predict the specimen force due to inertial oscillations generated in the system. These effects have been replicated with simple one degree of freedom mass-stiffness models [6]. It is the intention of the authors to apply alternative methods that rely on the monitoring of the crack length histories to obtain the specimen force [7]. This however falls out of the scope of the current investigation. Due to the above-mentioned, stress wave signal histories gathered from strain gauge stations (1) and (2) located at distances of $d_{1}=215 \mathrm{~mm}$ and $d_{2}=2155 \mathrm{~mm}$ from the specimen surface are shown as experimental outputs (see Figure 4).
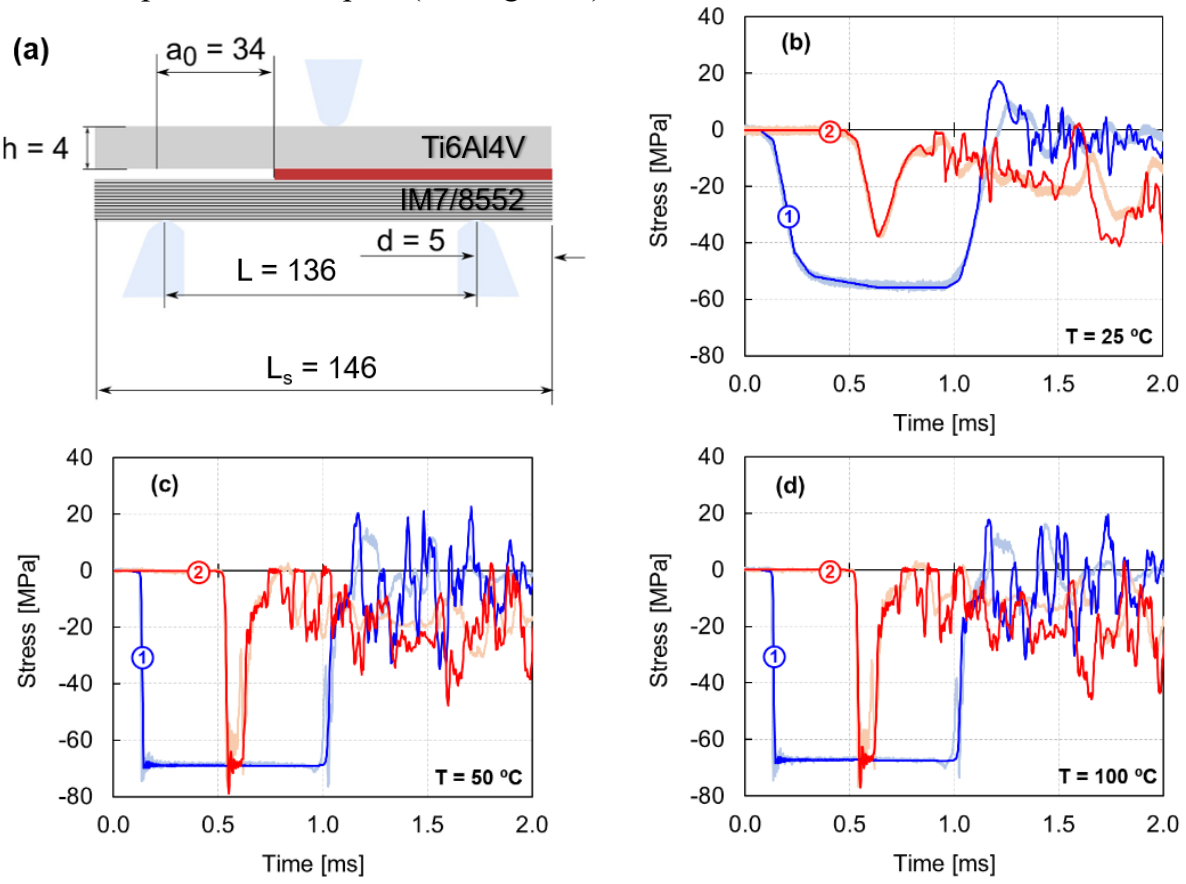

Figure 4. (a) Comparison of stress wave signal results for experiments (light) and simulations (dark) for temperatures of (b) $25^{\circ} \mathrm{C}$, (c) $50{ }^{\circ} \mathrm{C}$ and (d) $100{ }^{\circ} \mathrm{C}$. 
To validate the CZM presented in the previous section, FE models of the experimental setup shown in Figure 4 were generated and run in the ABAQUS/Explicit nonlinear solver. The model included the input bar, the end support modelled as a rigid surface, and the multimaterial specimen. The Titanium was assumed to be isotropic linear elastic and the CFRP laminate was discretised with 32 layers of orthotropic linear elastic material with the local orientations as indicated in the layup sequence. The material properties can be found in [4]. The adhesive layer was discretised using cohesive elements, while the rest employed 8-node reduced integration elements. The stress wave histories obtained from elements located at distances $d_{1}$ and $d_{2}$ from the specimen surface are plotted against the experimental readings for the three temperatures in Figure 4. In these we can observe that the signals are in reasonable agreement and the trends are being correctly represented. However, as mentioned before on top of the oscillations created due to the geometry and inertia of the system, we have to add the artificial numerical damping typically used in explicit time integration FE solvers. As emphasised before, other approaches to obtain the force histories will be explored in the future to strengthen our confidence in the predictive capabilities of the CZM.

\section{Concluding remarks}

A new set of data that includes mode II traction-separation response of the AF 163-2OST adhesive for three thicknesses $(0.1,0.3$ and $0.5 \mathrm{~mm})$ and three temperatures $(25,50$ and 100 ${ }^{\circ} \mathrm{C}$ ) at high strain rates has been presented. These tests have been used to calibrate a tabular temperature-dependent version of the CZM presented in [2]. High rate ENF tests at several temperatures have been carried out in a SHPB to validate the predictive capabilities of such model through FE simulations. The validation results suggest that alternative direct specimen force measurements that do not contain such a strong influence of the system inertia effects could be beneficial to further validate the proposed CZM.

The authors would like to acknowledge Rolls-Royce plc, for their continuing support through the Solid Mechanics University Technology Centre at the University of Oxford.

\section{References}

1. M. Banea et al., Effect of temperature on tensile strength and mode I fracture toughness of a high temperature epoxy adhesive, Journal of Adhesion Science and Technology, 26.7 (2012)

2. M. Lißner, E. Alabort, H. Cui, R. Rito, B.R.K. Blackmand, N. Petrinic., Experimental characterisation and numerical modelling of the influence of bondline thickness, loading rate and deformation mode on the response of ductile adhesive interfaces, Journal of the Mechanics and Physics of Solids, 130, 349-369, (2019)

3. Ruiz, C., Mines, R.A.W. The Hopkinson pressure bar: an alternative to the instrumented pendulum for Charpy tests. Int J Fract 29, 101-109 (1985)

4. M. Lißner, B. Erice, E. Alabort, D.Thomson, H.Cui, C.Kaboglu, B.R.K. Blackman, M. Gude, N.Petrinic, Multi-material adhesively bonded structures: Characterisation and modelling of their rate-dependent performance, Composites Part B: Engineering, 195, 108077 (2020)

5. Jiang, F., and Vecchio, K. S. (August 5, 2009). Hopkinson Bar Loaded Fracture Experimental Technique: A Critical Review of Dynamic Fracture Toughness Tests, ASME. Appl. Mech. Rev; 62(6): 060802 (2009)

6. Dutton, A.G., Mines, R.A.W. Analysis of the Hopkinson Pressure Bar loaded Instrumented Charpy Test using an inertial modelling technique. Int J Fract 51, 187-206 (1991).

7. M. Lißner, E. Alabort, B. Erice, H. Cui, B. R. K. Blackman, N. Petrinic, On the dynamic response of adhesively bonded structures, International Journal of Impact Engineering, 138, 103479 (2020) 